\title{
Short-Term Power Load Forecasting Based on EMD-Grey Model
}

\author{
Dong Jun, Wang Pei ${ }^{*}$, Dou Xihao \\ School of Economics and Management, North China Electric Power University, Beijing, China \\ Email address: \\ dongjun624@126.com (Dong Jun),1608156465@qq.com (Wang Pei),2268728128@qq.com (Dou Xihao) \\ ${ }^{*}$ Corresponding author
}

\section{To cite this article:}

Dong Jun, Wang Pei, Dou Xihao. Short-Term Power Load Forecasting Based on EMD-Grey Model. American Journal of Electrical Power and Energy Systems. Vol. 7, No. 4, 2018, pp. 42-49. doi: 10.11648/j.epes.20180704.11

Received: July 30, 2018; Accepted: August 14, 2018; Published: September 4, 2018

\begin{abstract}
With the issuance of "electricity reform No. 9 document" in 2015, a new round of power system reform in China has been continuously pushed forward. With the gradual development of the pilot spot market in various provinces, the importance of load forecasting to the various main bodies of the spot power market has been constantly revealed. In order to improve the accuracy of short-term load forecasting in the spot market, and better highlight the randomness, periodicity and related trend of load fluctuation, this paper proposes a short-term load forecasting based on grey model and the EMD combination model, predict the future 24-hour load. In other words, $\operatorname{GM}(1,1)$ is used to predict the residual value sequence of EMD decomposition. In order to ensure the stability of the residual value sequence, improve the accuracy of the prediction and improve the effect of short-term load forecasting. Combined with MATLAB tools, the combined prediction model was simulated and verified by using the America PJM power market load data. The comparison results of the combined model with the single $\mathrm{GM}(1,1)$ and $\mathrm{GM}(1,2)$ respectively show that the combined model can significantly improve the accuracy of load forecasting compared with the traditional grey model method, providing the method guidance for load forecasting to better participate in the demand response under the new market environment.
\end{abstract}

Keywords: Load Forecasting, EMD, Grey Model, Combination Model

\section{Introduction}

With the rapid development of the smart grid technology and the improvement of the power market construction, the load forecasting of power system, which is the basis of the power grid management, is of great significance to the formulation of economic and rational power generation plan, unit combination, power grid planning, dispatching operation and so on. It can better guarantee the reliable development of national industry, agriculture and Commerce, the accurate load forecasting data also provides reliable support for the construction of intelligent power grid. The power system load forecasting [1] is based on the historical data of power load, economy and society to explore the influence of the change law of power load historical data on the future load, so as to make a scientific research on the future power load. In recent years, the economy of our country has developed rapidly, the demand for electricity is increasing gradually in all walks of life, due to the power itself cannot be a large amount of storage, if regardless of supply and demand and mass production, will lead to a drop in quality of power supply or the waste of energy, so accurate short-term load forecasting method to become one of the most important aspects of the smart grid security health run.

At present, many domestic and foreign scholars have studied the short-term load forecasting, the existing main methods are the time series method [2], the exponential smoothing method [3], the wavelet analysis method [4], the support vector machine (SVM) method [5], the regression analysis [6], the trend analysis method [7] and so on, each method has its advantages and disadvantages, and has a certain scope of application, generally need according to the actual situation to choose the appropriate model to forecast. The most commonly used models are time series model and neural network. Artificial neural network is used to simulate the intelligent operation and good performance of the brain nerve, is often used in the study of short-term load forecasting, 
such as Li Long, Wei Jing, Li Canbing, Cao Yijia, Song Junying and Fang Baling [8], use artificial neural network to predict the parameters of the load model at the maximum and minimum load time, and analyze the sensitivity between the load model parameters and the load forecasting results, they have mastered their relationship and influence degree; Zhu Xuexiong [9] through the analysis of power load, daily temperature and historical load, proposed a set of short-term residential load prediction model based on artificial neural network, which improved the reliability of the prediction. Artificial neural network algorithm also has some defects, its convergence speed is slow, it is easy to fall into local minimum and so on, which hinders the further application and development of artificial neural network in the field of research. Zhao Haiqing [10] proposed a grey prediction model based on the accumulation method, which simplified the calculation process of the grey model, reduced the morbidity of the grey prediction, and overcame the defects of using the traditional grey model for prediction. Zhang Bing [11] introduced the random forest regression model into the short-term load prediction of power system, proposed a short-term load prediction method based on similar days and random forest regression model, and improved the accuracy of load prediction; Li Chuntao, Li Xiaocong, Yuan Hui, Qui Hao and Luo Hongliang [12] optimized the selection of background value parameters in the grey model by introducing an improved cuckoo optimization algorithm, which significantly improved the prediction accuracy of the grey model. The grey model is easy to use and can be applied to both the soft science of load forecasting and the hard science of industrial process forecasting and control. However, there are some problems in the prediction process of grey model. Among them, $\operatorname{GM}(1,1)$ is only applicable to situations where the signal is monotonous and cannot accurately predict the load curve with periodic or random fluctuations. When $\operatorname{GM}(1,2)$ is modeled, the reference sequence must have strong correlation with the main sequence. How to determine the reference sequence reasonably is a difficult problem to be considered in the modeling of GM $(1,2)$. In general, a single grey model is difficult to effectively reflect the authenticity of the prediction results, and generally needs to be combined with other models. Deng Ao and Jin Min [13] decomposed the loads through EMD, extracted the time-scale characteristics of each characteristic value, and carried out the de-redundancy of the characteristic value, which highlighted the randomness, periodicity and related trends of load fluctuations. Liu Xiaozhe [14] predicted the stable data generated by Empirical Mode Decomposition (EMD) through the grey markov model, and solved the problem that the grey markov method could not predict too many time periods for large fluctuating loads while improving the prediction accuracy. There are many examples of using neural networks, grey models and other models to predict load trends at home and abroad, but there is no case of combining EMD with GM $(1,1)$. According to the monotonous increasing characteristic of the residual value sequence of EMD, this paper combines EMD and GM $(1,1)$, first decomposes the load curve of the electricity market for one week before PJM, and then decomposes the residual value sequence with GM $(1,1)$ to fit the trend, preserving the original volatility of the load, and providing decision-making basis for future planning or scheduling of power grid.

\section{Theoretical Basis}

\subsection{Empirical Mode Decomposition Method}

Empirical mode decomposition [15] (EMD) is an adaptive and effective non-linear and non-stationary time-frequency data analysis method proposed by Huang et al., NASA. This method is a new signal analysis method, which decomposes non-stationary signals into several eigenmode components (IMF) in sequence according to the fluctuation or trend of different scales and smooths the signals to reduce the interference of characteristic information between signals. The basic idea of EMD decomposition is that if the maximum or minimum number of an original data sequence is two (or more than two) more than the number of upper-or lower-crossing zeros, the data sequence needs to be stabilized.

For a given signal $x(t) \in R$, the EMD process is as follows:

(1) Find all local extreme points of $x(t)$;

(2) For maximum and minimum value points, the maximum value envelope function and minimum value envelope function of the signal are respectively established by spline interpolation and denoted as $e_{\max }(t)$ and $e_{\min }(t)$;

(3) Calculate the mean value function of upper and lower envelope functions:

$$
e_{m}(t)=\frac{e_{\max }(t)+e_{\min }(t)}{2}
$$

(4) Calculate the difference function: $h(t)=x(t)-e_{m}(t)$

(5) Use $h(t)$ instead $x(t)$, repeat the above steps until the obtained envelope average tends to zero, and the first IMF component $c_{1}(t)$ is obtained. The low frequency signal in the original signal is $x_{1}(t)=x(t)-c_{1}(t)$. The ith IMF component $c_{i}(t)$ can be obtained by repeating the above process successively, when the difference function $x_{n}(t)$ is a constant function or a monotone function, the original signal can be expressed by these IMF functions $c_{j}(t), j=1,2, \ldots n$, and the trend function:.

$$
x(t)=\sum_{i=1}^{n} c_{j}(t)+r_{n}(t)
$$

\subsection{Grey Prediction Modeling Method}

\subsubsection{GM (1,1) Model}

GM $(1,1)$ is the most widely used and simplest grey model, which consists of a differential equation consisting of a single variable. Its essence is by accumulating the original data sequence and weakening the influence of random disturbance factors. The exponential curve is used to simulate the exponential growth law. The prediction process is as follows:

(1). The original sequence of historical loads is:

$$
x^{(0)}=\left(x^{(0)}(1), x^{(0)}(2), x^{(0)}(3), \ldots,\left(x^{(0)}(n)\right)\right.
$$


(2). Cumulative generation of the original sequence:

$x^{(1)}=\left(x^{(1)}(1), x^{(1)}(2), x^{(1)}(3), \ldots,\left(x^{(1)}(n)\right)\right.$, In the form,

$$
x^{(1)}(i)=\sum_{j=1}^{i} x^{(0)}(j),(j=1,2,3, \ldots, n)
$$

(3). The first order differential equation model is constructed:

$$
\frac{d x^{(1)}}{d t}+a x^{(1)}=u
$$

after solving a and $u$, the average value of $J$ and $j+1$ times is obtained:

$$
\frac{1}{2}\left[x^{(1)}(j+1)+x^{(1)}(j)\right]
$$

and the generating matrix is as follows:

$$
\left(\begin{array}{c}
x^{(0)}(2) \\
x^{(0)}(3) \\
x^{(0)}(4) \\
\cdot \\
\cdot \\
x^{(0)}(n)
\end{array}\right)=\left(\begin{array}{c}
-\frac{1}{2}\left[x^{(1)}(1)+x^{(1)}(2)\right] 1 \\
-\frac{1}{2}\left[x^{(1)}(2)+x^{(1)}(3)\right] 1 \\
-\frac{1}{2}\left[x^{(1)}(3)+x^{(1)}(4)\right] 1 \\
\cdot \\
\cdot \\
. \\
-\frac{1}{2}\left[x^{(1)}(n-1)+x^{(1)}(n)\right] 1
\end{array}\right)\left(\begin{array}{l}
a \\
u
\end{array}\right)
$$

Brief account:

$$
\begin{gathered}
Y_{n}=B A, \text { among them } Y_{n}=\left(\begin{array}{c}
x^{(0)}(2) \\
x^{(0)}(3) \\
x^{(0)}(4) \\
\cdot \\
\cdot \\
\cdot \\
x^{(0)}(n)
\end{array}\right), A=\left(\begin{array}{l}
a \\
u
\end{array}\right), \\
B=\left(\begin{array}{c}
-\frac{1}{2}\left[x^{(1)}(1)+x^{(1)}(2)\right] 1 \\
-\frac{1}{2}\left[x^{(1)}(2)+x^{(1)}(3)\right] 1 \\
-\frac{1}{2}\left[x^{(1)}(3)+x^{(1)}(4)\right] 1 \\
. \\
. \\
. \\
-\frac{1}{2}\left[x^{(1)}(n-1)+x^{(1)}(n)\right] 1
\end{array}\right)
\end{gathered}
$$

The prediction model is as follows:

$$
\begin{gathered}
\hat{x}^{(0)}(\mathrm{j}+1)=\hat{x}^{(1)}(\mathrm{j}+1)-\hat{x}^{(1)}(\mathrm{j}) \\
=\left(1-e^{\hat{a}}\right)\left(x^{(0)}(1)-\frac{\hat{u}}{\hat{a}}\right) e^{-\hat{a} j} \\
(j=1,2,3 \ldots n)
\end{gathered}
$$

\subsubsection{GM $(1, n)$ Model}

When there are $\mathrm{n}$ variables in the power system, the prediction process is as follows:

(1). The original sequence of historical loads is:

$$
x_{1}^{(0)}=\left(x_{1}^{(0)}(1), x_{1}^{(0)}(2), x_{1}^{(0)}(3), \ldots, x_{1}^{(0)}(n)\right)
$$

the target sequence of the system is: $x_{1}{ }^{(0)}(i=1)$, the sequence of the related factors of the system is

$$
x_{1}^{(0)}(i=1,2,3, \ldots n) \text {. }
$$

(2). Cumulative generation of the original sequence: $x_{1}^{(1)}=\left(x_{1}^{(1)}(1), x_{1}^{(1)}(2), x_{1}^{(1)}(3), \ldots, x_{1}^{(1)}(n)\right)$, In the form, $x_{1}^{(1)}(t)=\sum_{k=1}^{t} x_{1}^{(0)}(k)$, the $\operatorname{GM}(1, \mathrm{n})$ model is a first-order grey differential equation with $\mathrm{n}$ variables.

(3). Constructing a first order differential equation model:

$\frac{d x_{1}^{(1)}}{d t}+a x_{1}^{(1)}=b_{2} x_{2}^{(1)}+b_{3} x_{3}^{(1)}+b_{4} x_{4}^{(1)}+\cdots+b_{n} x_{n}^{(1)}$

the generating matrix is as follows:

$$
\left(\begin{array}{c}
x_{1}^{(0)}(2) \\
x_{1}^{(0)}(3) \\
x_{1}^{(0)}(4) \\
\cdot \\
\cdot \\
x_{1}^{(0)}(n)
\end{array}\right)=\left(\begin{array}{c}
-z(2) x_{2}^{(1)}(2) \ldots . x_{N}^{(1)}(2) \\
-z(3) x_{2}^{(1)}(3) \ldots . x_{N}^{(1)}(3) \\
-z(4) x_{2}^{(1)}(4) \ldots . x_{N}^{(1)}(4) \\
. . \\
. . \\
\ldots \\
-z(n) x_{2}^{(1)}(n) \ldots . x_{N}^{(1)}(n)
\end{array}\right)\left(\begin{array}{c}
a \\
b_{2} \\
b_{3} \\
b_{4} \\
\cdot \\
\cdot \\
\cdot \\
b_{n}
\end{array}\right)
$$

Brief account:

$$
\begin{gathered}
Y_{n}=B A \text {, among them } Y_{n}=\left(\begin{array}{c}
x_{1}^{(0)}(2) \\
x_{1}^{(0)}(3) \\
x_{1}^{(0)}(4) \\
\cdot \\
\cdot \\
\cdot \\
x_{1}^{(0)}(n)
\end{array}\right), A=\left(\begin{array}{c}
a \\
b_{2} \\
b_{3} \\
b_{4} \\
\cdot \\
\cdot \\
\cdot \\
b_{n}
\end{array}\right), \\
B=\left(\begin{array}{c}
-z(2) x_{2}^{(1)}(2) \ldots x_{N}^{(1)}(2) \\
-z(3) x_{2}^{(1)}(3) \ldots . x_{N}^{(1)}(3) \\
-z(4) x_{2}^{(1)}(4) \ldots . x_{N}^{(1)}(4) \\
\ldots \\
. . \\
\ldots \\
-z(n) x_{2}^{(1)}(n) \ldots . x_{N}^{(1)}(n)
\end{array}\right)
\end{gathered}
$$

The prediction model is as follows:

$$
\hat{x}_{1}^{(0)}(\mathrm{j}+1)=\hat{x}_{1}^{(1)}(\mathrm{j}+1)-\hat{x}_{1}^{(1)}(\mathrm{j})(j=0,1,2, \ldots)
$$




\section{Combination Model Prediction Method}

The grey model has the characteristics of small calculation and high accuracy. In order to improve the accuracy of the prediction, the grey model and EMD are combined with the new prediction model EMD-grey model to improve the prediction ability of the model for the short-term load. The main idea is to use EMD to decompose the historical load curve, and then use GM $(1,1)$ to predict the decomposed value sequence to improve the stability of the load curve. Finally, the predicted residual sequence is fitted to the trend of the IMF component, and the authenticity of the short-term load curve is reduced, making the prediction result more accurate. Through EMD and GM $(1,1)$, the accuracy of traditional grey model prediction is rarely seen in load forecasting. The main prediction process is as follows:

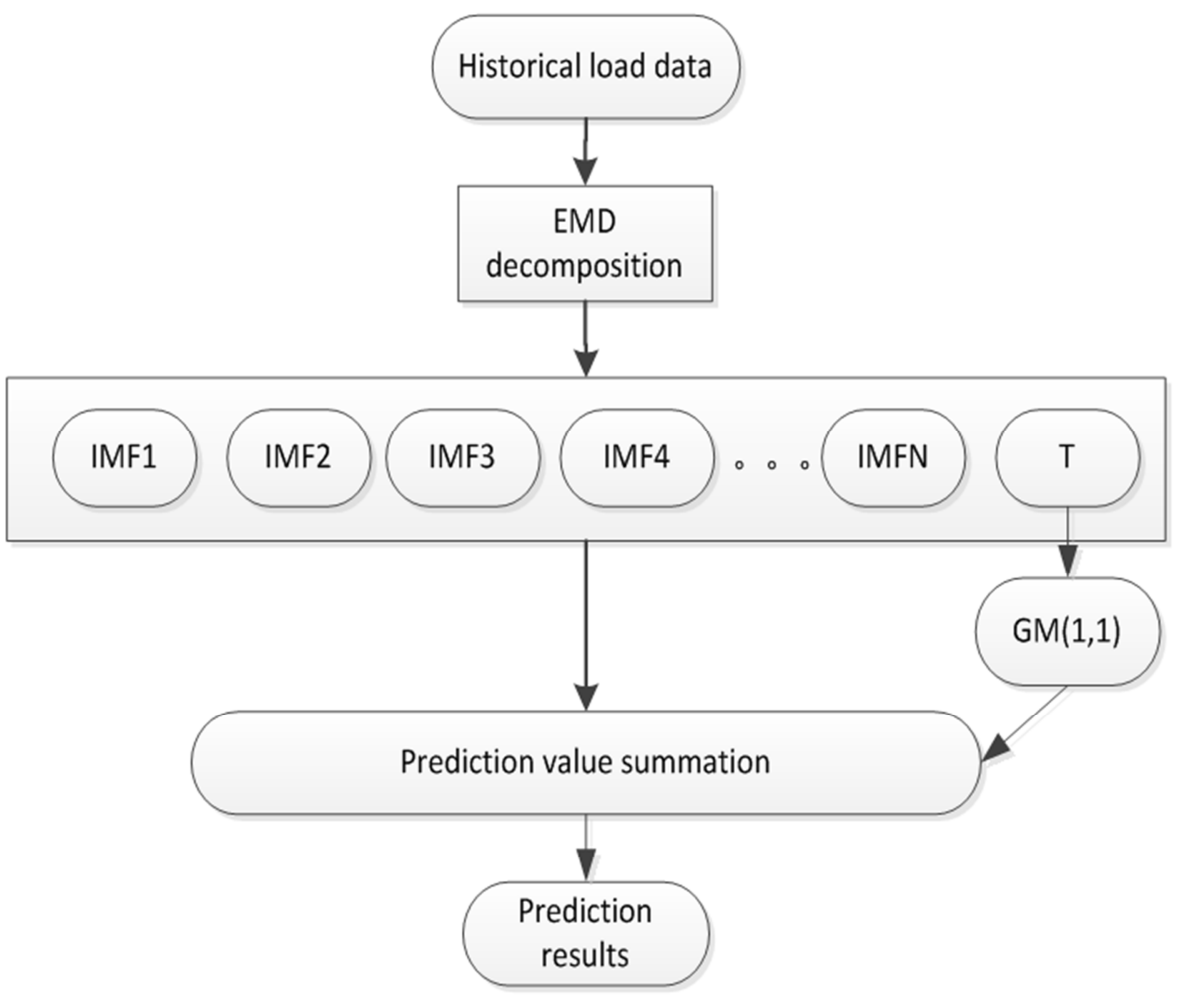

Figure 1. EMD-grey model load forecasting framework.

The specific steps of EMD-grey model prediction are as follows:

(1). Input the time series $x(t)$ of the original signal historical load into the EMD model, and the original signal is decomposed into several IMF and residuals $r_{n}(t)$;

(2). The residual error $r_{n}(t)$ is predicted by $\operatorname{GM}(1,1)$, and the corresponding residual trend $\mathrm{T}$ is obtained;

(3). The predicted values of each IMF and T are fitted to get the final load forecast data;

(4). The mean absolute percentage error is a prediction error index expressed by relative percentage, and it is one of the important indicators to measure the prediction accuracy, Its expression is:

$$
M A P E=\frac{1}{K} \sum_{i=1}^{K} \frac{\left|x_{i}^{(0)}(j)-\tilde{x}_{i}^{(0)}(j)\right|}{\tilde{x}_{i}^{(0)}(j)}
$$

The average deviation rate is the average of the absolute deviation of all individual observations and arithmetic mean values. Its expression is:

$$
\delta_{M A P E}=\frac{1}{K} \sum_{i=1}^{K} \frac{\left|x_{1}^{(0)}(j)-\tilde{x}_{1}^{(0)}(j)\right|}{\bar{x}_{1}}
$$

In the form: MAPE represents an average absolute percentile error, MAPE indicates the average deviation rate, $\mathrm{K}$ indicates the number of data, $x_{1}^{(0)}(j)$ represents the real data value, $\tilde{x}_{1}^{(0)}(j)$ indicates the value of prediction data, $\bar{x}_{1}$ indicates the average value of real data, and the smaller the $\delta_{M A P E}$ value, the higher the accuracy of model fitting.

\section{Simulation Contrast}

\subsection{A Brief Introduction to an Example}

The United States PJM is the largest wholesale market in the world, the electricity trading business, the power grid planning, the technical support system are relatively mature, all kinds of systems are perfect. The PJM power market load can better reflect the supply and demand characteristics of the power market and the change of the market structure. At present, many provinces in our country have taken PJM as the spot market, 
adopt the PJM electricity market of load data has certain forward-looking. Therefore, considering the above factors and data storage habits, the difficulty of load acquisition, and the feasibility of verification of the proposed method, the historical load data of PJM from February 8th to February 14th and its corresponding historical load data from February 1st to February 7 th are simulated and predicted for the sample data, figure 2 to the original load sequence diagrams

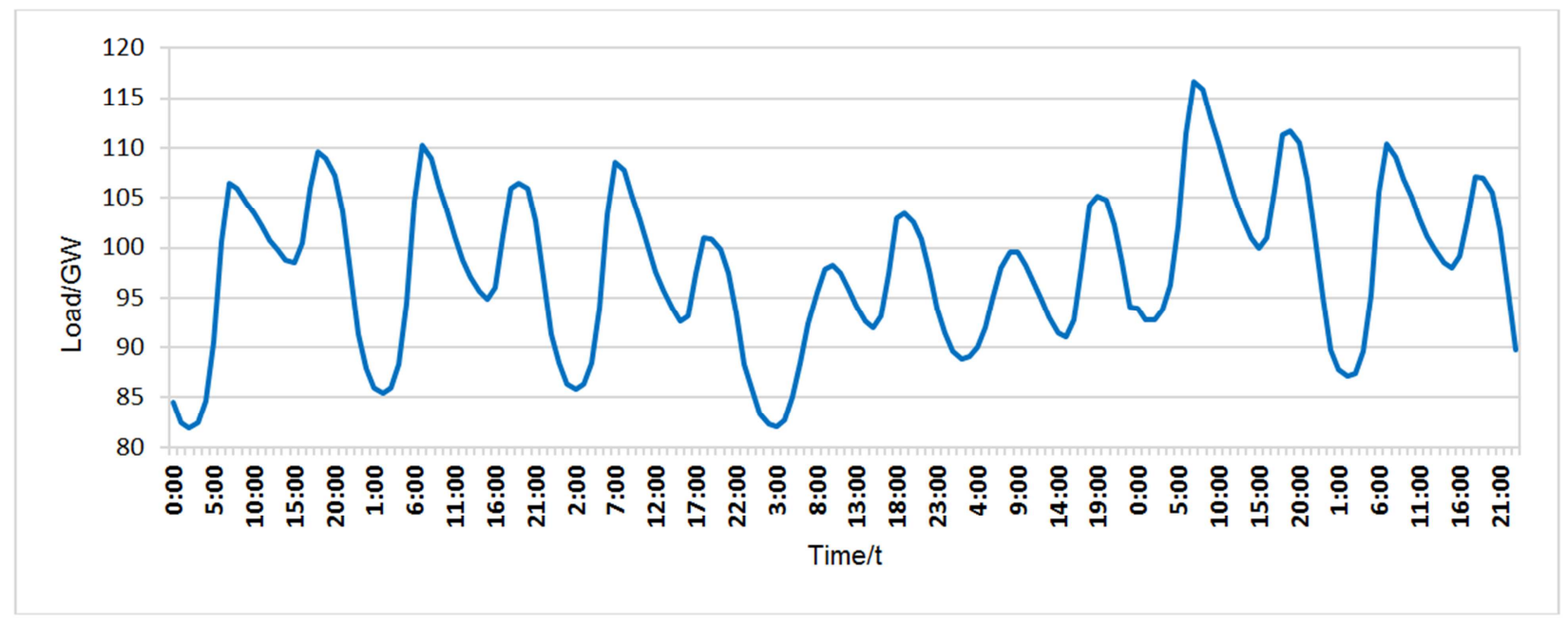

Figure 2. PJM spot market one week historical load.

\subsection{EMD Decomposition Results}

To decompose sample data with EMD, as shown in Figure 3, EMD decomposes the original load sequence into four groups of IMF and T. From Figure 4, it can be seen that the signal after decomposition has a different trend of change, and the steady data will help to reduce the deviation of the grey model prediction, and the residual data can be carried out with GM $(1,1)$. Prediction fitting.
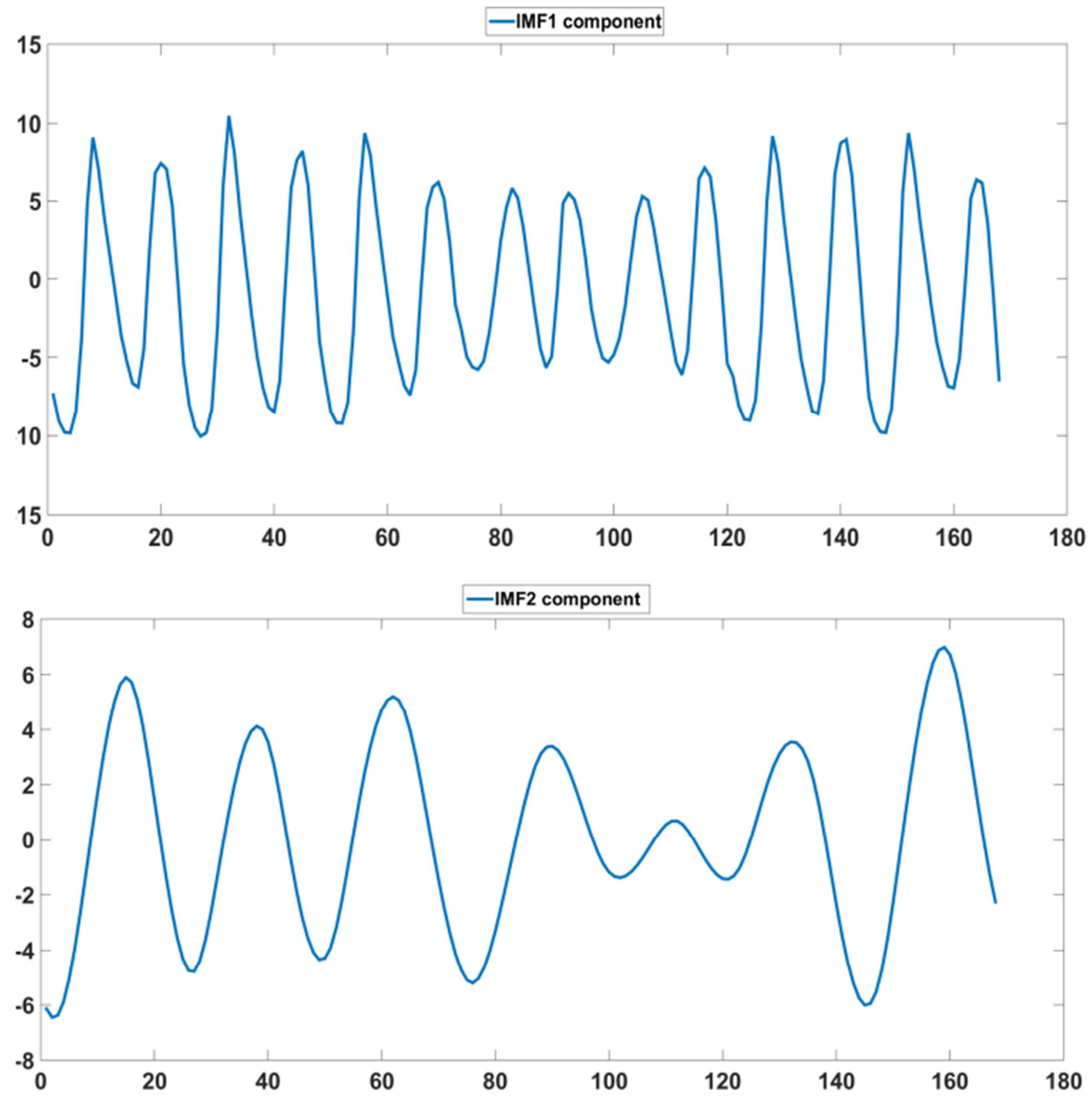

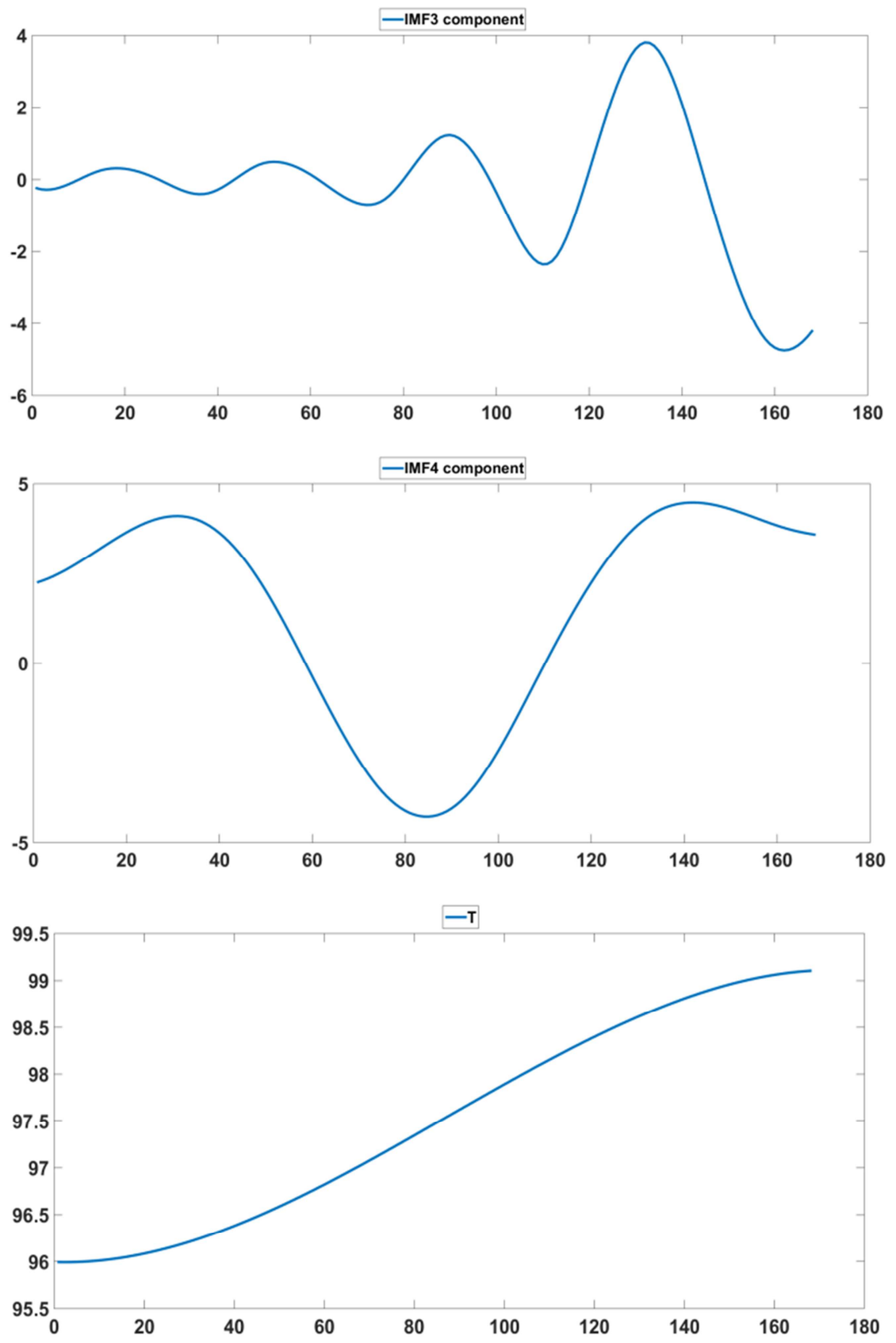

Figure 3. EMD load decomposition results.

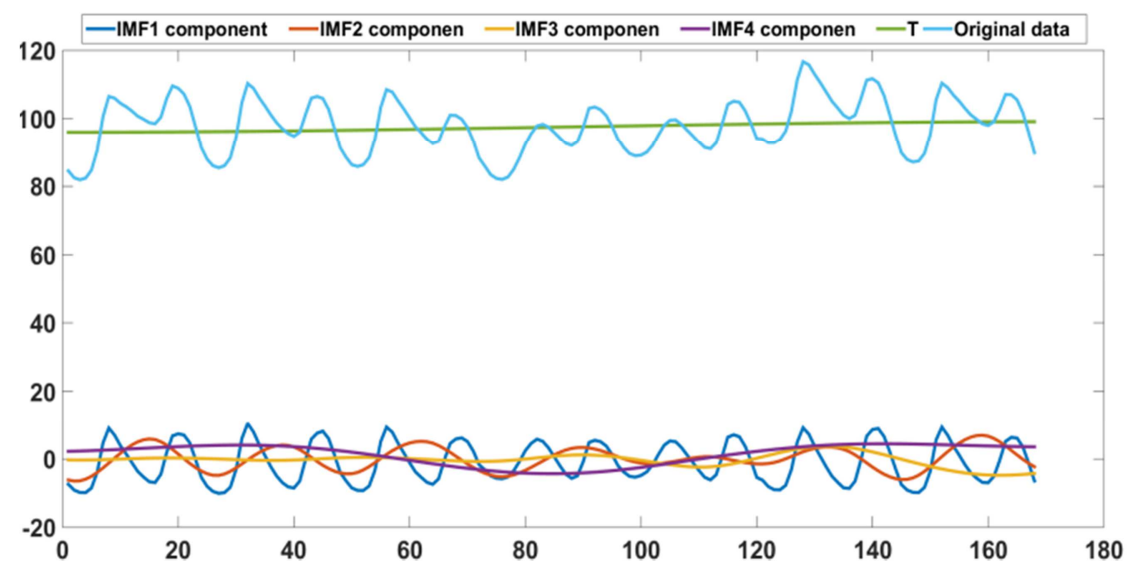

Figure 4. Comparison of the original load series decomposition with the original load sequence. 


\subsection{Comparison and Analysis of Model Results}

To compare the accuracy of the model, a comparison group was established. GM $(1,1)$ was used to predict the undecomposed original sequence as model 1; GM $(1,2)$ was used to predict the undecomposed original sequence as model 2; The load will be decomposed using EMD and then simulated using GM $(1,1)$ as model 3 . The difference between model 3 and model 1 model 2 is that model 3 preprocesses the original load sequence of PJM. By decomposing the original data, we get a curve which is slower than the original curve, which does not change the characteristics of the original series, and makes the prediction result more precise. MAPE and are used to evaluate the performance of the model respectively, and the results of three models are compared in a certain period. The results of the test forecast are like table 1, and the comparison between the three models and the original load is shown in figure 5.

Table 1. Comparison of historical data with three schemes in a certain period of time/GW.

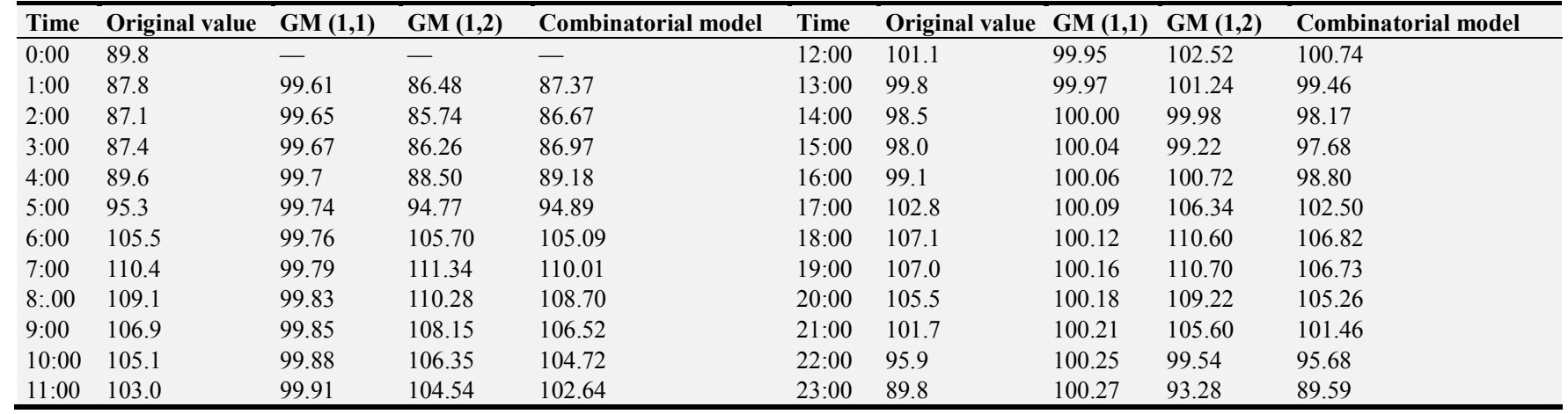

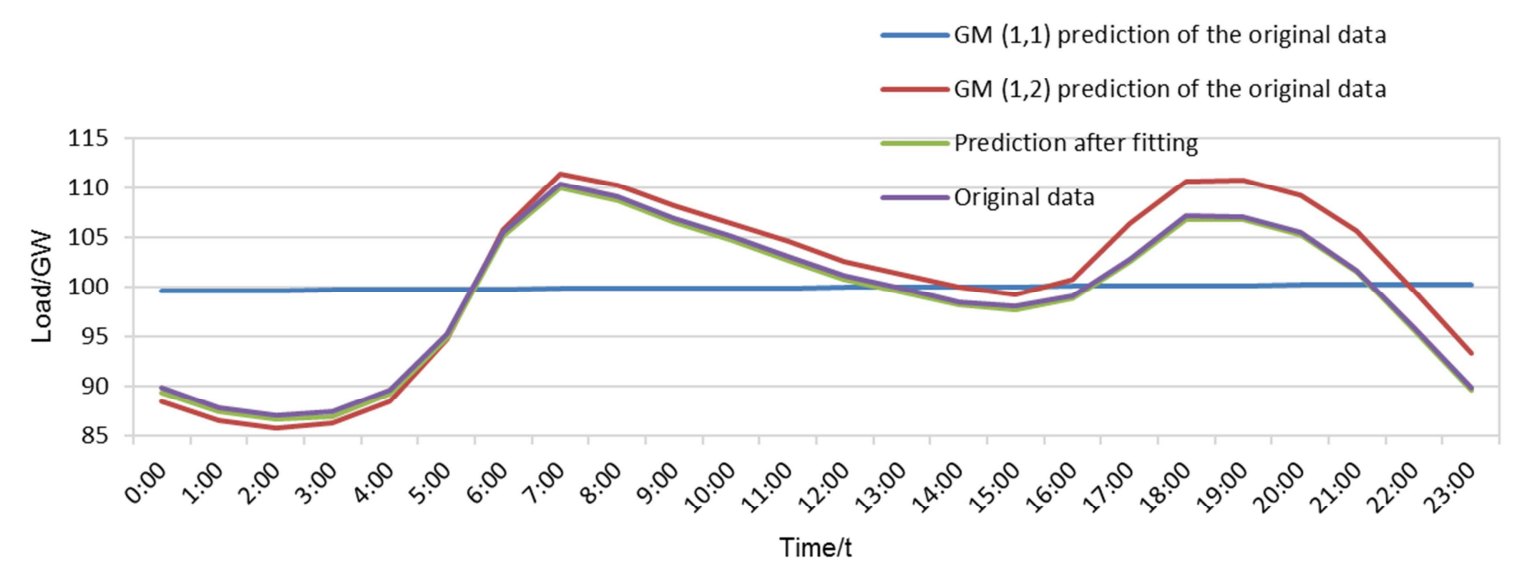

Figure 5. Comparison of historical data with three schemes in a certain period of time.

Table 2. Error Comparison between Three Models.

\begin{tabular}{llll}
\hline Time & Model1_MAPE & Model2_MAPE & Model3_MAPE \\
\hline 0:00 & 0.098212492 & 0.0147 & 0.005037754 \\
1:00 & 0.108387443 & 0.0150 & 0.00500796 \\
2:00 & 0.114238560 & 0.0153 & 0.004989608 \\
3:00 & 0.116455482 & 0.0148 & 0.004987441 \\
$4: 00$ & 0.113425168 & 0.0143 & 0.004924263 \\
$5: 00$ & 0.101940264 & 0.0128 & 0.004822995 \\
6:00 & 0.095597096 & 0.0113 & 0.004694236 \\
$7: 00$ & 0.096937869 & 0.0109 & 0.004555336 \\
$8: 00$ & 0.096484535 & 0.0109 & 0.00445365 \\
9:00 & 0.093896672 & 0.0110 & 0.004361966 \\
$10: 00$ & 0.090111767 & 0.0110 & 0.004292958 \\
$11: 00$ & 0.085179772 & 0.0113 & 0.004225331 \\
12:00 & 0.079512540 & 0.0115 & 0.004172794 \\
$13: 00$ & 0.073954538 & 0.0117 & 0.004115934 \\
$14: 00$ & 0.070024236 & 0.0119 & 0.004068592 \\
\hline
\end{tabular}

\begin{tabular}{llll}
\hline Time & Model1_MAPE & Model2_MAPE & Model3_MAPE \\
\hline $15: 00$ & 0.066922211 & 0.0120 & 0.004020505 \\
$16: 00$ & 0.063549978 & 0.0122 & 0.003962403 \\
$17: 00$ & 0.061523625 & 0.0134 & 0.003902949 \\
$18: 00$ & 0.061954821 & 0.0143 & 0.003836837 \\
$19: 00$ & 0.062271616 & 0.0153 & 0.00377074 \\
$20: 00$ & 0.061835083 & 0.0162 & 0.003701357 \\
$21: 00$ & 0.059700251 & 0.0171 & 0.003639765 \\
$22: 00$ & 0.058991175 & 0.0180 & 0.003582395 \\
$23: 00$ & 0.060883963 & 0.0188 & 0.003528622 \\
$\delta_{M A P E}$ & 0.0645 & 0.0433 & 0.0023 \\
\hline
\end{tabular}

From the comparison between the load sequence predicted by the three models in table 1 and figure 5 and the original load sequence, it can be seen that model 1 is accurate only when the load curve is linearly changing, and there is a large deviation in the prediction of the load curve with volatility. 
Model 2 in load forecast of volatile, at every turning point and the fluctuation of extreme value point basic outliers can be generated from the original course load history, therefore in the short-term load volatile situation, predicted results cannot reflect the authenticity of the original load sequence, mainly related to the selection of relevant factors listed. The GM $(1,1)$ prediction of the historical load after EMD decomposition is closer to the historical data than that of the load series prediction without decomposition in the same period. The predicted results can well reflect the volatility of the original sequence at the turning point or fluctuation point of the historical load data, and are closer to the real value of the historical load series. From the error comparison of the three models, it can be found that the load prediction error of the combined model is lower than that of the grey model without decomposition, indicating that the combined model is more accurate.

\section{Conclusion}

In this paper, according to the characteristics of the volatility of the short-term load in the market, and according to the characteristics of the EMD time-frequency analysis, a combination prediction model of the EMD-grey model is proposed. The model is simulated with the historical load data of the electric power market of PJM in the United States as the sample, and the simulation is compared with the traditional grey model prediction. The results show that the accuracy of the load sequence predicted only by using grey model is not high, and it is easily affected by many factors, so only a single grey model can not effectively reflect the characteristics of historical load sequence. The combined prediction model proposed in this paper is more accurate than the direct grey model forecasting, and the prediction is more accurate. It can reflect the volatility of the original sequence more authentically, reduce the generation of jump points, and is more operable. Therefore, it can be explained that under the background of the reform of the power system, the EMD-grey model can predict the load in the case of less historical data, and can meet the demand of real-time load forecasting under the demand response under the spot market. The application of the portfolio model will provide a certain prediction reference for the market players in the new energy market.

\section{References}

[1] Fei Xi. Short-term load prediction of power system based on linear time series mathematical model $[\mathrm{J}]$. China high-tech zone, 2018(14).
[2] Yuan S, Yang L, Shang B, Li X, Zhang H. Short term power network gateway load forecasting algorithm based on ARMR model[C]// Information Technology, Networking, Electronic and Automation Control Conference, IEEE. IEEE, 2016: 497-501.

[3] Tan Fenglei, Zhang Zhaojun, Zhu Chao, Zhang jun. Study on an improved exponential smoothing load forecasting method [J]. Power demand side management, 2016, 18(6): 22-26.

[4] Xie Beimin, Zhao Xuesong. Study on power load prediction method based on improved wavelet analysis [J]. Science and technology innovation and application, 2016(36): 207-207.

[5] Xie min, Deng Jialiang, Ji Xiang, Liu mingbo. A method to predict the cooling load of support vector machines based on the optimization of information entropy and variable precision rough set [J]. Grid technology, 2017, 41(1): 210-214.

[6] Cheng Chao. Study on improved forecasting method of monthly power sales based on time series method and regression analysis [D]. Chongqing university, 2016.

[7] Silva PRN, Carvalho AP, Gabbar HA, Vieira P, Costa CT. Fault Diagnosis in Transmission Lines Based on Leakage Current and Qualitative Trend Analysis[C]// International Conference on Promising Electronic Technologies. 2017: 87-92.

[8] Li Long, Wei Jing, Li Canbing, Cao Yijia, Song Junying, Fang Baling. Load model prediction based on artificial neural network [J]. Acta Electrotechnical Sinica, 2015, 30(8): 225-230.

[9] Zhu Xuexiong. Forecasting research of short-term residential load based on artificial neural network [J]. Science and technology innovation and application, 2017(23): 0-20.

[10] Zhao Haiqing. Grey model based on accumulation method and its application in power load prediction [J]. China electric power, 2016(s1): 94-95.

[11] Zhang Bing, Zhou Buxiang, Shi Min, Wei Jinxiao. Short-term load prediction based on grey correlation analysis and random forest regression model [J]. Hydro-electric energy science, 2017(4): 203-207.

[12] Li Chuntao, Li Xiaocong, Yuan Hui, Qui Hao, Luo Hongliang. Short-term load forecasting based on improved grey model [J]. electric switch, 2017, 55 (2): 11-13.

[13] Deng Ao, Jin Min. Emd-based time scale feature extraction and its application in short-term power load prediction [J]. Computer application research, 2018(10): 1-6.

[14] Liu Xiaozhe. Research on power load forecasting based on EMD-Grey Markov model [D]. HeFei University of Technology, 2013.

[15] Huang N E. New method for nonlinear and nonstationary time series analysis: empirical mode decomposition and Hilbert spectral analysis [C]// Proc of SPIE: The International Society for Optical Engineering, 2000: 197-209. 